Supporting Information

\title{
Transfer-free PZT Thin Films for Flexible Nanogenerators Derived from Single-step Modified Sol-gel Process on 2D mica
}

\author{
Shiyuan Liu ${ }^{1}$, Deng Zou ${ }^{1}$, Xinge $\mathbf{Y u}^{2^{* *}}$, Zuankai Wang ${ }^{1 *}$, Zhengbao Yang ${ }^{13^{* *}}$ \\ 1 Department of Mechanical Engineering, City University of Hong Kong, Hong Kong, China \\ 2 Department of Biomedical Engineering, City University of Hong Kong, Hong Kong, China \\ 3 Shenzhen Research Institute of City University of Hong Kong, Shenzhen 518057, China
}

*Correspondence to: X.Y. (xingeyu@cityu.edu.hk), Z.W. (zuanwang@cityu.edu.hk); Z.Y.

(zb.yang@cityu.edu.hk). 
a

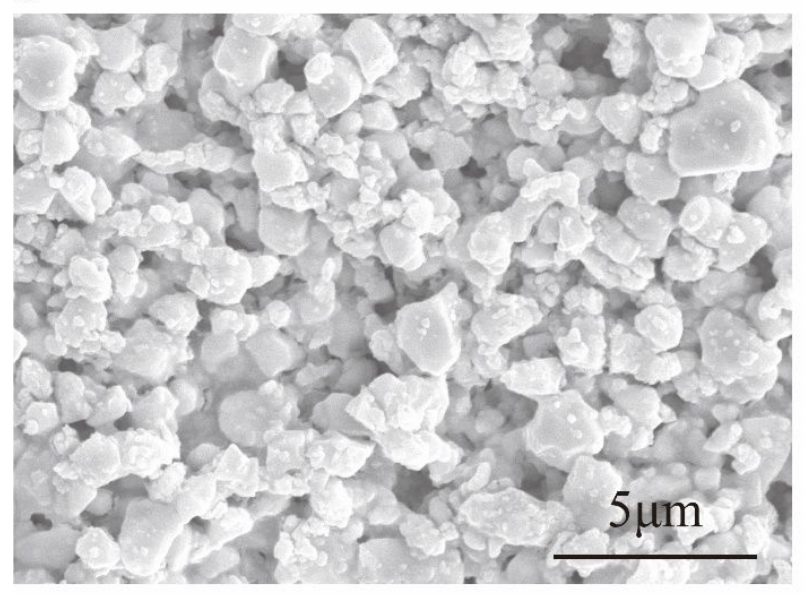

$\mathrm{b}$

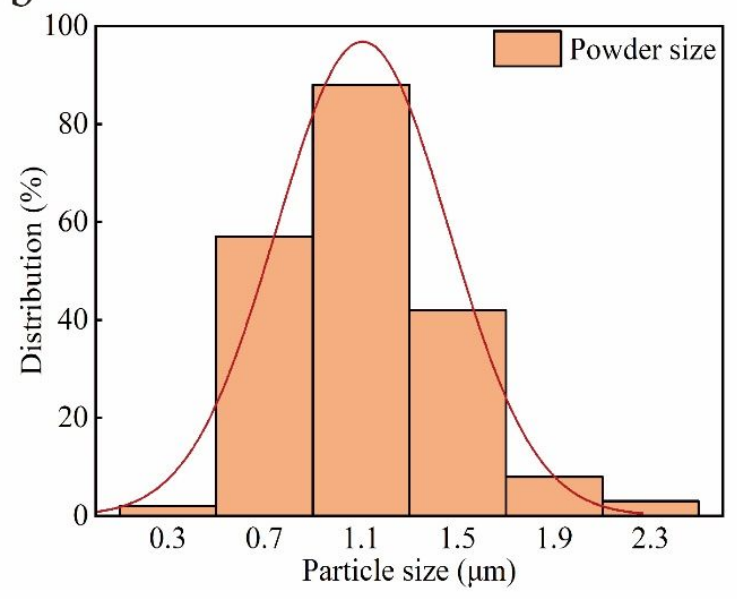

Figure S1. The characteristic of the powder-based PZT thin film. (a) The top surface SEM image of the powder-based PZT thin film. (b) The particle size distribution of the PZT powder in the powder-based film. 
(a)
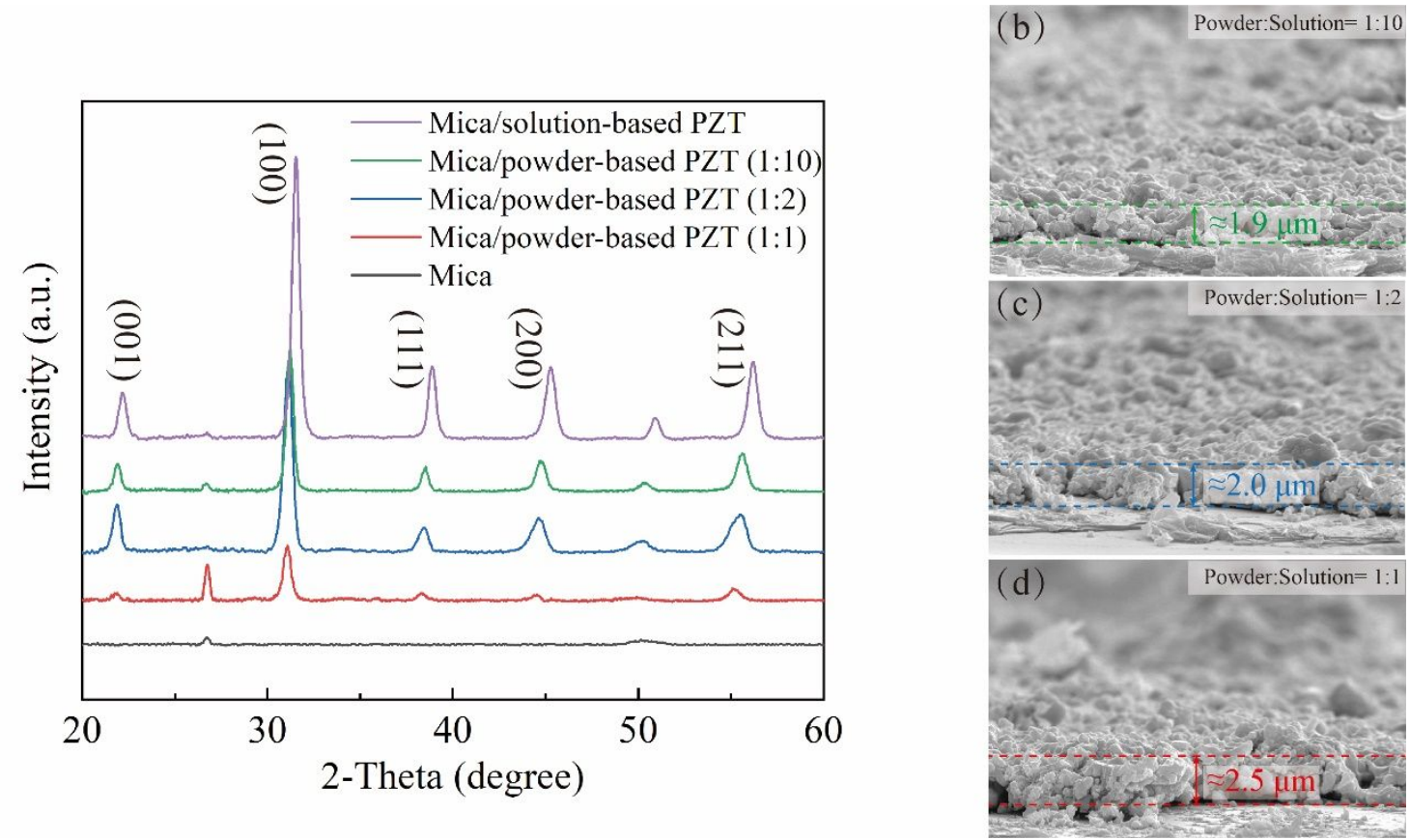

Figure S2 The properties of PZT films with three different weight percentage of powder. (a) The XRD pattern comparison among mica, mica/powder-based PZT with three different weight ratio and mica/solution-based PZT. (b-d) The cross-sectional SEM image of three types of powderbased PZT films. 


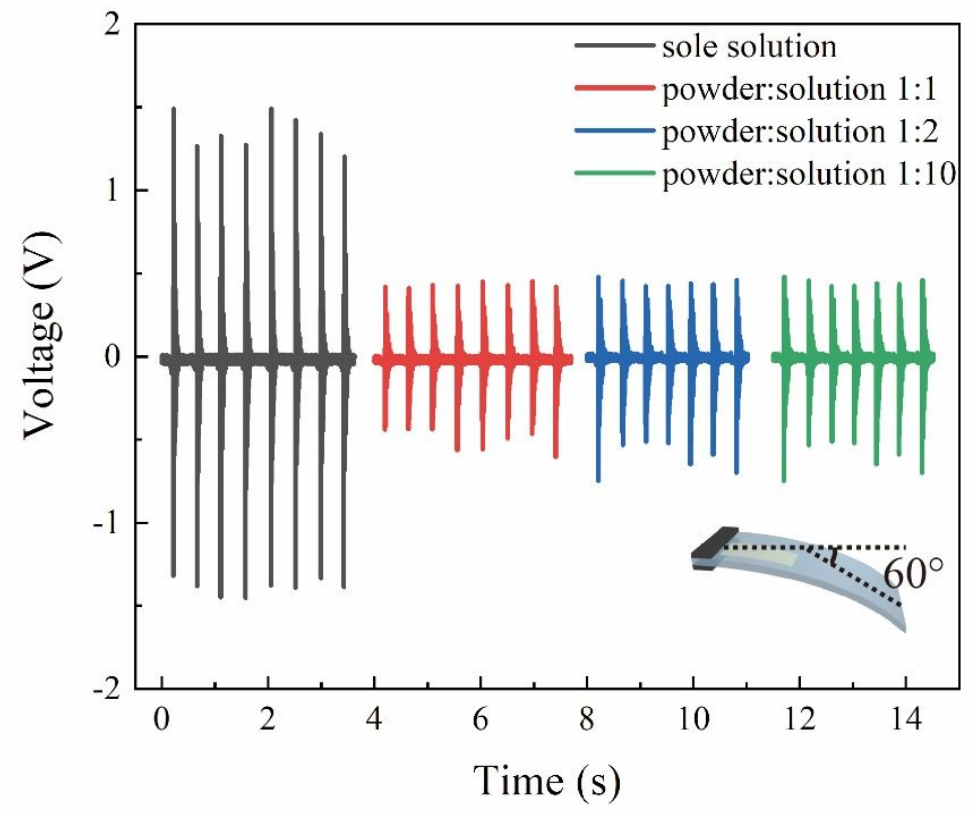

Figure S3 The comparison of the piezoelectric outputs from bending-releasing mode of three different powder weight percentage of PZT films. 
(a)

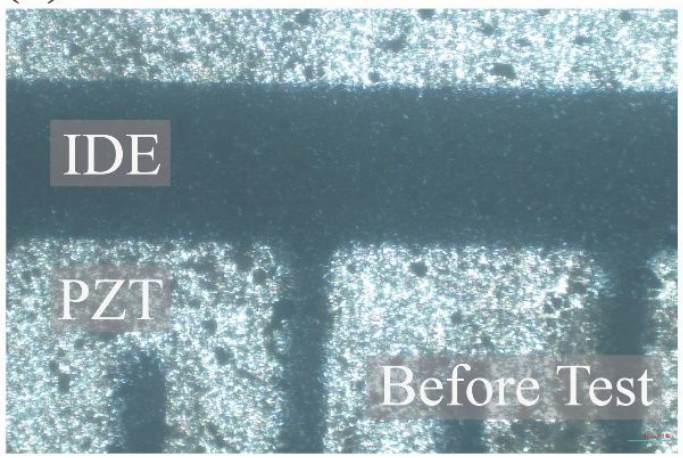

(b)

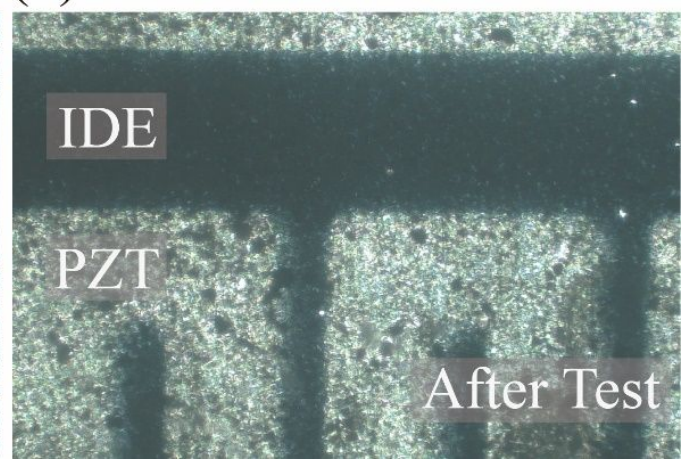

Figure S4 The optical microscope images of the surface of the powder-based PENG. (a) The surface morphology of the as-prepared powder-based PENG. (b) The surface morphology of the powder-based PENG after 50,000 tapping cycles. 
Table S1. The basic properties of Fluorophlogopite Mica.

\begin{tabular}{|c|c|}
\hline Body Resistivity & $10^{15}-10^{16} \Omega / \mathrm{cm}$ \\
\hline Surface Resistivity & $10^{11}-10^{13} \Omega$ \\
\hline Intensity of Electric Breakdown & $185-238 \mathrm{kV} / \mathrm{mm}$ \\
\hline Melting Point & $1375^{\circ} \mathrm{C}$ \\
\hline Max. Service Temperature & $1100^{\circ} \mathrm{C}$ \\
\hline
\end{tabular}

